UDC: 517.9

\title{
Numerical Solution of Linear and Higher-order Delay Differential Equations using the Coded Differential Transform Method
}

\author{
G. Methi ${ }^{\mathrm{a}}$, A. Kumar \\ Manipal University Jaipur, \\ Department of Mathematics \& Statistics, Manipal University Jaipur, Rajasthan, India \\ E-mail: ${ }^{a}$ girirajmethi@gmail.com, ${ }^{\mathrm{b}}$ anil.digit@gmail.com
}

The aim of the paper is to obtain a numerical solution for linear and higher-order delay differential equations (DDEs) using the coded differential transform method (CDTM). The CDTM is developed and applied to delay problems to show the efficiency of the proposed method. The coded differential transform method is a combination of the differential transform method and Mathematica software. We construct recursive relations for a few delay problems, which results in simultaneous equations, and solve them to obtain various series solution terms using the coded differential transform method. The numerical solution obtained by CDTM is compared with an exact solution. Numerical results and error analysis are presented for delay differential equations to show that the proposed method is suitable for solving delay differential equations. It is established that the delay differential equations under discussion are solvable in a specific domain. The error between the CDTM solution and the exact solution becomes very small if more terms are included in the series solution. The coded differential transform method reduces complex calculations, avoids discretization, linearization, and saves calculation time. In addition, it is easy to implement and robust. Error analysis shows that CDTM is consistent and converges fast. We obtain more accurate results using the coded differential transform method as compared to other methods.

Keywords: delay differential equations, coded differential transform method, numerical solution, mathematica

Citation: Computer Research and Modeling, 2019, vol. 11, no. 6, pp. 1091-1099.

C 2019 Giriraj Methi, Anil Kumar This work is licensed under the Creative Commons Attribution-NoDerivs 3.0 Unported License. To view a copy of this license, visit http://creativecommons.org/licenses/by-nd/3.0/ or send a letter to Creative Commons, PO Box 1866, Mountain View, CA 94042, USA. 


\section{Introduction}

Delay differential equations (DDEs) involve the rate of change of time dependent process determined by the present state as well as the previous state. It has found applications in biology [Nelson, Perelson, 2002], environment [Herrera, 2013], control theory [Raji et al.,2013] and [Milton, 2015], etc. There are various numerical methods available to solve DDEs. In [Ismail, Suleiman, 2001] the Runga-Kutta method is used to solve stiff DDEs, and the Newton divided difference interpolation is applied to approximate the delay argument. In [Evans, Raslan, 2004] DDEs are investigated using the approximate series Adomian decomposition method with Mathematica. The authors of [Shekari, Dehghan, 2008] applied the homotopy perturbation method to DDEs to obtain a numerical solution. They compared the series solution with the exact solution without using Mathematica.

Recently the differential transform method (DTM) has been used by many researchers to obtain solutions of differential equations. The authors of [Raslan, Sheer, 2013] applied series DTM to obtain solutions of nonlinear systems of partial differential equations without using any numerical code. They compared their numerical solution with an exact solution and the Adomian decomposition method. [Benhammouda, Hector, 2004] investigated some nonlinear delay differential equations with the multistep differential transform method to obtain an analytical solution. They converted DDEs into ordinary differential equations, which are then solved using DTM without using any code. In this context we propose a new method, the coded differential transform method (CDTM), to solve delay differential equations.

The main motivation of the paper is to develop the coded differential transform method for solving linear and higher-order DDEs. The series solution differential transform method is coded using Mathematica software and applied to linear and higher-order DDEs to obtain an approximate series solution. The CDTM has the advantage of reducing complex calculations and saves computation time and allows a better accuracy as compared to existing methods.

\section{Analysis of the differential transform method}

The differential transform of the kth derivative of function $u(x)$ is given by

$$
U(k)=\frac{1}{k !}\left[\frac{d^{k} u(x)}{d x^{k}}\right]_{x=x_{0}},
$$

where $u(x)$ is the original given function and $U(k)$ are transformed functions. The inverse differential transform of $U(k)$ is given by

$$
u(x)=\sum_{k=0}^{\infty} U(k)\left(x-x_{0}\right)^{k} .
$$

From equations (1) and (2),

$$
u(x)=\sum_{k=0}^{\infty} \frac{\left(x-x_{0}\right)^{k}}{k !}\left[\frac{d^{k} u(x)}{d x^{k}}\right]_{x=x_{0}} .
$$

Some results for the differential transform method.

1. If $y(x)=\frac{d^{n} u(x)}{d x^{n}}$, then $Y(k)=(k+1)(k+2)(k+3) \ldots(k+n) U(k+n)$.

2. If $y(x)=x^{n}$, then $Y(k)=\delta(k-n)$, where $\delta(k-n)= \begin{cases}1, & k=n, \\ 0, & k \neq n\end{cases}$

3. If $y(x)=e^{\alpha x}$, then $Y(k)=\frac{\alpha^{k}}{k !}$. 
4. If $y(x)=u(x-\alpha)$ where $\alpha>0$, then $Y(k)=\sum_{i=k}^{N}(-\alpha)^{i-k}\left(\begin{array}{c}i \\ k\end{array}\right) U(i)$.

5. If $y(x)=u_{1}(a x) u_{2}(b x)$, then $Y(k)=\sum_{i=0}^{k}(a)^{i}(b)^{k-i} U_{1}(i) U_{2}(k-i)$.

\section{The coded differential transform method}

\section{Example 1}

For $\left[\mathrm{k}=0, \mathrm{k} \leq 4, \mathrm{k}=\mathrm{k}+1, \operatorname{Print}\left[-1000 \mathrm{Y}[\mathrm{k}]-\sum_{i=k}^{5}\left(-N[\log [999], 10)^{i-k}\right.\right.\right.$ Binomial $[i, k] Y[i]$ $-(k+1) Y[k+1]]]$

\section{Example 2}

$\mathrm{k}=0 ; \operatorname{Print}\left[(\mathrm{k}+1)(\mathrm{k}+2) \mathrm{Y}[\mathrm{k}+2]-\mathrm{Y}[\mathrm{k}]+4 \sum_{i=0}^{k}\left(\frac{1}{2}\right)^{k} \operatorname{Cos}\left[\frac{i P i}{i !}\right] Y[k-i]\right]$

\section{Example 3}

For $\left[\mathrm{k}=0, \mathrm{k} \leq 6, \mathrm{k}=\mathrm{k}+1, \operatorname{Print}\left[-\mathrm{Y}[\mathrm{k}]+\sum_{i=k}^{8}\left(-N[P i, 10)^{i-k} \operatorname{Binomial}[i, k] Y[i]\right.\right.\right.$ $-2(k+1)(k+2) Y[k+2]]]$

\section{Example 4} $+2]]]$

For $\left[\mathrm{k}=0 ; \mathrm{k} \leq 8, \mathrm{k}=\mathrm{k}+1, \operatorname{Print}\left[\sum_{i=k}^{10}\left(-N[P i, 10)^{i-k}\right.\right.\right.$ Binomial $[i, k] Y[i]-(k+1)(k+2) Y[k+$

\section{Test Examples}

We apply the coded differential transform method to delay differential equations to obtain numerical solutions.

\section{Example 1}

Consider DDE [Roth, 1980]

$$
y^{\prime}(t)=-1000 y(t)-y(t-\ln 999) .
$$

The initial condition is $y(0)=1$.

The exact solution is

$$
y(t)=e^{-t} .
$$

Applying DTM to equation (4), we get

$$
(k+1) y(k+1)=-1000 y(k)-\sum_{i=k}^{N}(-\ln 999)^{i-k}\left(\begin{array}{c}
i \\
k
\end{array}\right) y(i) .
$$

Now, taking $N=5$ and $k=0,1,2,3,4$ in equation (6), we obtain a system of linear equations as

$$
\begin{gathered}
5.9067548 y(1)-47.703262 y(2)+329.47473 y(3)-2275.6012 y(4)+15717.019 y(5)=1001, \\
-1001 y(1)+11.81351 y(2)-143.10978 y(3)+1317.8989 y(4)-11378.006 y(5)=0, \\
-1001 y(2)+17.720264 y(3)-286.21957 y(4)+3294.7473 y(5)=0 \\
-1001 y(3)+23.627019 y(4)-477.03262 y(5)=0 \\
-1001 y(4)+29.533774 y(5)=0
\end{gathered}
$$


Solving equations (7)-(11), we get

$$
\begin{gathered}
y(1)=-0.735557 ; \quad y(2)=0.214654 ; \quad y(3)=-0.0312327 ; \\
y(4)=0.00193405 ; \quad y(5)=0.0655516 .
\end{gathered}
$$

Now, the series solution is given by

$$
y(t)=1-0.735557 t+0.214654 t^{2}-0.0312327 t^{3}+0.00193405 t^{4}+0.0655516 t^{5}+\ldots
$$

The numerical solution obtained in equation (13) and the exact solution are plotted for different values of $y$ and $t$ shown in Fig. 1 .

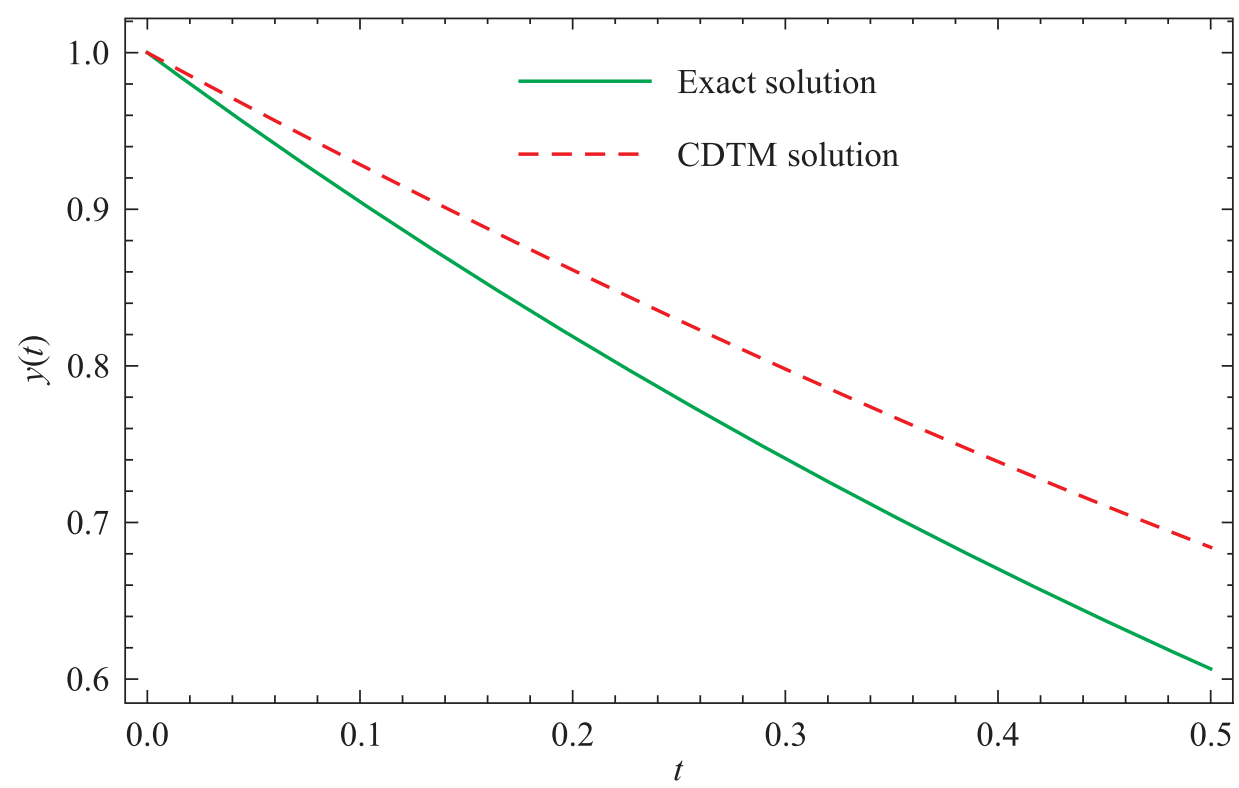

Figure 1. Cdtm and an exact solution plotted for different values of $t$ for Example 1

Numerical illustrations in Fig. 1 show that the CDTM solution approximates the exact solution for specific values of $t$, but when we increase the value of $t$, the difference between CDTM solution and the exact solution increases.

\section{Example 2}

Consider DDE

$$
y^{\prime \prime}(t)-y(t)+\eta(t) y\left(\frac{t}{2}\right)=0, \quad 0 \leq t \leq 2 \pi
$$

where

$$
\eta(t)=\frac{4 \sin (t)}{(2-2 \cos (t))^{1 / 2}} .
$$

The initial conditions are

$$
y(0)=0, \quad y^{\prime}(0)=1 .
$$

The exact solution is

$$
y(t)=\sin (t) .
$$

After simplification of equation (14) we get

$$
y^{\prime \prime}(t)-y(t)+4 \cos \left(\frac{t}{2}\right) y\left(\frac{t}{2}\right)=0 .
$$


Applying DTM to equation (18) and equation(16), we get

$$
\begin{gathered}
(k+1)(k+2) Y(k+2)-Y(k)+4 \sum_{i=0}^{k}\left(\frac{1}{2}\right)^{k} \frac{\cos \left(\frac{i \pi}{2}\right)}{i !} Y(k-i)=0, \\
Y(0)=0, \quad Y(1)=1 .
\end{gathered}
$$

Putting $k=0,1,2,3, \ldots$ in equation (19) and using equation (20), we get the following series components:

$$
Y(2)=0, \quad Y(3)=-\frac{1}{3 !}, \quad Y(4)=0, \quad Y(5)=-\frac{1}{5 !} \ldots
$$

Now the series solution is given by

$$
y(t)=t-\frac{1}{3 !} t^{3}+\frac{1}{5 !} t^{5}-\frac{1}{7 !} t^{7}+\ldots
$$

The numerical solution obtained in equation (22) and the exact solution are plotted for different values of $y$ and $t$ shown in Fig. 2.



Figure 2. Cdtm and an exact solution plotted for different value of $t$ for Example 2

Numerical illustrations in Fig. 2 show that the CDTM solution approximates the exact solution for specific values of $t$, but we increase the value of $t$ and the difference between the CDTM solution and the exact solution decreases.

\section{Example 3}

Consider DDE

$$
y^{\prime \prime}(t)=-\frac{1}{2} y(t)+\frac{1}{2} y(t-\pi), \quad t \in[0, \pi] .
$$

The exact solution is

$$
y(t)=1-\sin t
$$

The initial conditions are

$$
y(0)=1, \quad y^{\prime}(0)=-1
$$


Applying DTM to equation (23), we get

$$
(k+1)(k+2) y(k+2)=-\frac{1}{2} y(k)+\frac{1}{2} \sum_{i=k}^{N}(-\pi)^{i-k}\left(\begin{array}{c}
i \\
k
\end{array}\right) y(i) .
$$

Now, if we take $N=8$ and $k=0,1,2,3, \ldots, 6$ in equation (26) and solve the system of linear equations, then the series solution is given by

$$
\begin{gathered}
y(t)=1-t-0.413198 t^{2}+0.150668 t^{3}+0.0632388 t^{4}+0.0160175 t^{5}+0.00213381 t^{6} \\
-0.000368748 t^{7}-0.0000493414 t^{8}+\ldots
\end{gathered}
$$

The numerical solution obtained in equation (27) and the exact solution are plotted for different values of $y$ and $t$ shown in Fig. 3.



Figure 3. Cdtm and an exact solution plotted for different values of $t$ for Example 3

Numerical illustrations in Fig.3 show that the CDTM solution approximates the exact solution for specific values of $t$, but we increase the value of $t$ and the difference between the CDTM solution and the exact solution increases.

\section{Example 4}

Consider DDE

$$
y^{\prime \prime}(t)=y(t-\pi), \quad t \in[0, \pi] .
$$

The exact solution is

$$
y(t)=\sin t .
$$

The initial conditions are

$$
y(0)=0, \quad y^{\prime}(0)=1 .
$$

Applying DTM to equation (28), we get

$$
(k+1)(k+2) y(k+2)=\sum_{i=k}^{N}(-\pi)^{i-k}\left(\begin{array}{c}
i \\
k
\end{array}\right) y(i) .
$$


Now, taking $N=10$ and $k=0,1,2, \ldots, 8$ in equation (31), we solve a system of linear equations and the series solution is given up to ten terms by

$$
\begin{gathered}
y(t)=t+0.29635 t^{2}-0.119779 t^{3}-0.0191354 t^{4}+0.00651269 t^{5}+0.000680791 t^{6} \\
-0.000151587 t^{7}-0.0000123138 t^{8}+.00000207178 t^{9}+0.000000200185 t^{10}+\ldots
\end{gathered}
$$

The numerical solution obtained in equation (32) and the exact solution are plotted for different values of $y$ and $t$ shown in Fig. 4.

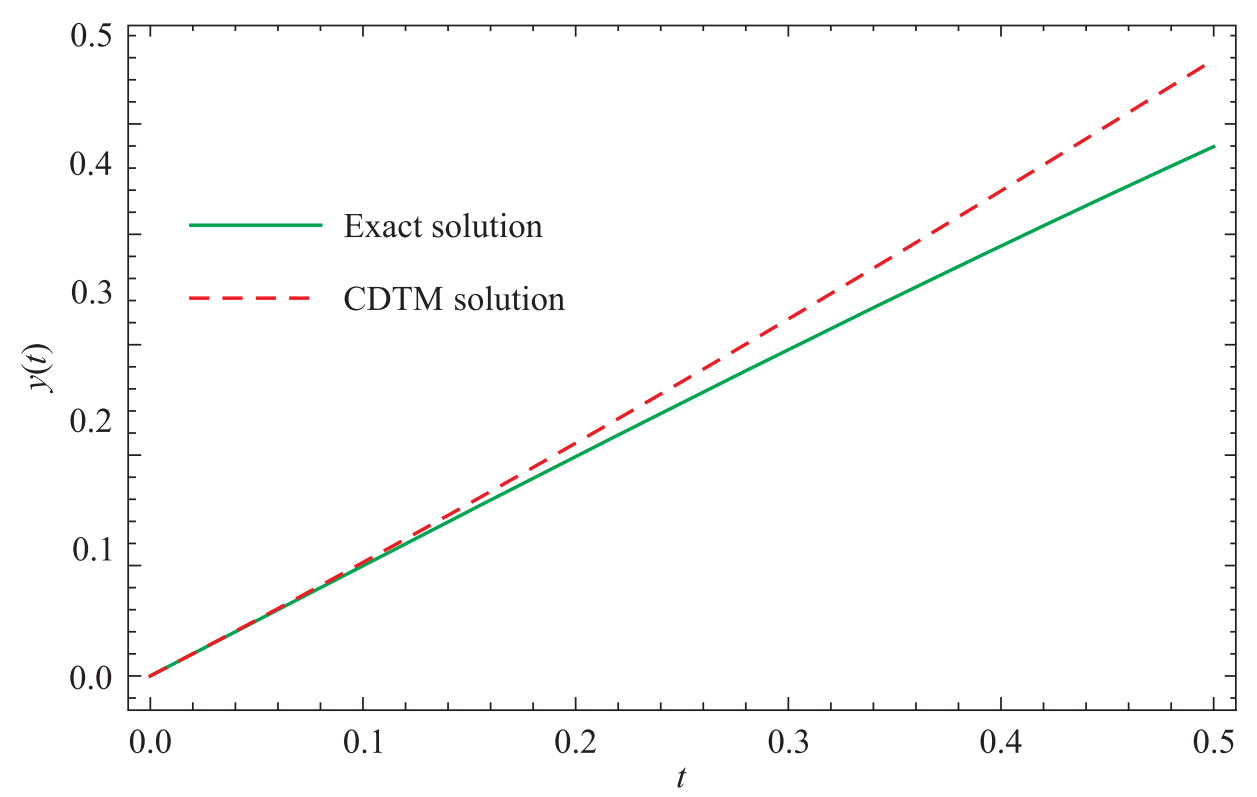

Figure 4. CDTM and an exact solution for different value of $t$ for Example 4

Numerical illustrations in Fig. 4 show that the CDTM solution approximates the exact solution for specific values of $t$, but we increase the value of $t$ and the difference between the CDTM solution and the exact solution increases.

\section{Error Analysis}

We have computed the error analysis for Examples 1, 2, 3 and 4 shown in Tables 1, 2, 3 and 4. For $t$ in the domain $0.05 \leq t \leq 0.50$ we have shown the difference between the CDTM solution and the exact solution. However, when we increase the value of $t$, the difference between the CDTM and the exact solution increases.

\section{Conclusion}

A new technique, the coded differential transform method (CDTM), is presented and applied to delay differential equations for an approximate solution. The difference between the CDTM and the exact solution is small for specific values of $t$ and it increases when the value of $t$ increases. The CDTM has the advantage of reducing complex calculations, saves computation time and allows better results to be obtained. 
Table 1. Absolute error $\left|y_{c d t m}-y_{\text {exact }}\right|$ in Example 1

\begin{tabular}{|c|c|}
\hline$t$ & $\left|y_{c d t m}-y_{\text {exact }}\right|$ \\
\hline 0.05 & 0.0125255 \\
\hline 0.10 & 0.023723 \\
\hline 0.15 & 0.0336887 \\
\hline 0.20 & 0.0425182 \\
\hline 0.25 & 0.0503094 \\
\hline 0.30 & 0.0571652 \\
\hline 0.35 & 0.0631963 \\
\hline 0.40 & 0.0685237 \\
\hline 0.45 & 0.0732815 \\
\hline 0.50 & 0.0776196 \\
\hline
\end{tabular}

Table 3. Absolute error $\left|y_{c d t m}-y_{\text {exact }}\right|$ in Example 3

\begin{tabular}{|c|c|}
\hline$t$ & $\left|y_{c d t m}-y_{\text {exact }}\right|$ \\
\hline 0.05 & 0.00103459 \\
\hline 0.10 & 0.00414141 \\
\hline 0.15 & 0.00931706 \\
\hline 0.20 & 0.0165468 \\
\hline 0.25 & 0.0258036 \\
\hline 0.30 & 0.0370469 \\
\hline 0.35 & 0.0502223 \\
\hline 0.40 & 0.0652595 \\
\hline 0.45 & 0.0820724 \\
\hline 0.50 & 0.100557 \\
\hline
\end{tabular}

Table 2. Absolute error $\left|y_{c d t m}-y_{\text {exact }}\right|$ in Example 2

\begin{tabular}{|c|c|}
\hline$t$ & $\left|y_{\text {cdtm }}-y_{\text {exact }}\right|$ \\
\hline 0.05 & 0 \\
\hline 0.10 & 0 \\
\hline 0.15 & $2.77556 \cdot 10^{-17}$ \\
\hline 0.20 & $2.77556 \cdot 10^{-17}$ \\
\hline 0.25 & 0 \\
\hline 0.30 & 0 \\
\hline 0.35 & $2.22045 \cdot 10^{-16}$ \\
\hline 0.40 & $1.11022 \cdot 10^{-15}$ \\
\hline 0.45 & $4.996 \cdot 10^{-15}$ \\
\hline 0.50 & $1.95954 \cdot 10^{-14}$ \\
\hline
\end{tabular}

Table 4. Absolute error $\left|y_{c d t m}-y_{\text {exact }}\right|$ in Example 4

\begin{tabular}{|c|c|}
\hline$t$ & $\left|y_{c d t m}-y_{\text {exact }}\right|$ \\
\hline 0.05 & 0.000746616 \\
\hline 0.10 & 0.00300846 \\
\hline 0.15 & 0.0068163 \\
\hline 0.20 & 0.0121979 \\
\hline 0.25 & 0.0191781 \\
\hline 0.30 & 0.0277786 \\
\hline 0.35 & 0.0380177 \\
\hline 0.40 & 0.0499112 \\
\hline 0.45 & 0.0634711 \\
\hline 0.50 & 0.0787066 \\
\hline
\end{tabular}

\section{Acknowledgments}

The authors are very grateful to the editor and the reviewer for their constructive comments aimed at improving the paper.

\section{References}

Benhammouda B. Hector V.L. A new multi step technique with differential transform method for analytical solution of some nonlinear variable delay differential equations // Springer Plus. 2016. - Vol. 5. - P. 1-17.

Evans D.J. Raslan K.R. The Adomain decomposition method for Solving Delay Differential Equation // International Journal of Computer Mathematics. - 2004. - Vol. 1. - P. 1-6.

Herrera A. R. Chaos in delay differential equations with applications in population dynamics // Discrete and continuous dynamical systems. - 2013. - Vol. 33. - P. 1633-1644.

Ismail D., Suleiman M. B. Solving delay differential equations using interval wise partitioning by Runge - Kutta method // Applied mathematics and computation. - 2001. - Vol. 121. - P. 37-53. 
Milton J. G. Time delay and the control of biological systems: An Overview // IFAC-Papers Online. 2015. - Vol. 48(12). - P. 87-92.

Nelson P.W. Perelson A.S. Mathematical analysis of delay differential equation models of HIV-1 infection // Math. Biosci. - 2002. - Vol. 179. - P. 73-94.

Raji R.A. Oke M.O., Adewumi O.A. Optimal control of dealy differential equations // IOSR-JM. 2013. - Vol. 5. - P. 44-47.

Raslan K.R. Sheer Z.F. Differential Transform Method for solving nonlinear systems of partial differential equations // International journal of Physical Sciences. - 2013. - Vol. 8. - P. 18801884.

Roth M.G. Difference methods for stiff delay differential equations // Ph.D thesis. - University of Illinois at Urbana Champaign. - 1980.

Shekari F., Dehghan M. Solution of delay differential equations via a homotopy perturbation method // Mathematical and Computer Modelling. - 2008. - Vol. 48(3). - P. 486-498. 